\title{
Design and Experimental Verification of Double Star Sensor Bracket with Complex Space Angle for Microsatellites
}

\author{
Long Chen ${ }^{1}$, Yu Xu' ${ }^{1}$, Xiaoli li ${ }^{1}$, Tong Guo ${ }^{1}$, Cheng Zhu', Rui Liu ${ }^{1}$ \\ ${ }^{1}$ Innovation Academy for Microsatellites, Chinese Academy of Sciences, Shanghai 201203, China
}

\begin{abstract}
In this paper, a double star sensor bracket design with complex space angle is proposed in order to improve the precision of microsatellites attitude measurement. This bracket is lightweight and is easy to manufacture. Finite element simulation and mechanical experiments are carried out. It is shown that the bracket satisfies the strength and stiffness requirements of microsatellites.
\end{abstract}

\section{Introduction}

Star sensor is a high-precision measurement device for satellite attitude, and it plays an important role in satellite attitude measurement and control system ${ }^{[1-4]}$. Star sensor has the advantages of high measurement accuracy, driftless system, etc. Therefore, it is widely used in satellite attitude measurement systems ${ }^{[5-6]}$

Generally star sensor is installed to the structural panel of the satellite via a particular bracket in order to keep its orientation fixed ${ }^{[7]}$. At present, the main used star sensor bracket of satellites is generally designed as a single independent bracket or a simple space angle double-star sensor bracket. Since the star sensor is generally the most precise measuring device when the satellite is in orbit, it is impossible to correct the measured value directly. To ensure the practicality of the compact layout remote sensing microsatellite's star sensor and improve the measurement precision of microsatellite attitude, in this paper, a novel two-frame design is proposed to achieve better efficiency and accuracy. This design can ensure that the two star sensors are in similar thermal and mechanical conditions in space, through the results measured by the two star sensors, the measurement precision of satellite attitude might be improved by mutual calibration and mutual correction.

In this paper, a double-star sensor embedding system with complex space angles was designed for the satellite attitude control subsystem. Mechanical analysis and production process optimization were performed. Experiments are carried out in order to validate the effectiveness of our design.

\section{Model design}

\subsection{Design parameters}

The angles between Optical axis of the star sensor and the satellite coordinate system are given in Table 1. Each star sensor has weight of $0.22 \mathrm{~kg}$ and envelope of $69 \mathrm{~mm} \times 55 \mathrm{~mm} \times 110 \mathrm{~mm}$. After installation, the sinusoidal response magnification at $0-100 \mathrm{~Hz}$ should be no more than 1.5 times acceleration input, and the RMS random response magnification at $20-2000 \mathrm{~Hz}$ should be no more than 2.5 times RMS input. The envelopes of the two sensors should occupy minimum space and the maximum total weight is limited to $0.25 \mathrm{~kg}$. The deviation between star sensors and the main datum is less than $30^{\prime \prime}$ before and after the vibration experiment.

Table 1. The angel between the star sensors' optical axis and the satellite's coordinate system

\begin{tabular}{|c|c|c|c|}
\hline Items Direction & $\mathbf{X}$ & Y & $\mathbf{Z}$ \\
\hline Optical axis of star sensor A & $67.48^{\circ}$ & $130^{\circ}$ & $131.56^{\circ}$ \\
\hline Optical axis of star sensor B & $43.96^{\circ}$ & $70^{\circ}$ & $127.16^{\circ}$ \\
\hline
\end{tabular}

\subsection{Structural design}

With the above-mentioned parameters and constraints, a bracket model is designed and is shown in Figure 1. The material used is $2 \mathrm{~A} 12 \mathrm{~T} 4$ aluminum alloy, and the total weight is $0.245 \mathrm{~kg}$.

The detailed design procedures are: a) draw the optical axes of star sensor A and star sensor B from the origin of the datum coordinate; b) draw star-sensors' mounting surfaces; c) establish normal planes of the two mounting surfaces; d) stretch the mounting surface entity based on the normal palne; e) cut the entity based on the mounting surface of the star sensor; f) draw bracket mounting surface; g) lightweight design and process optimization. 


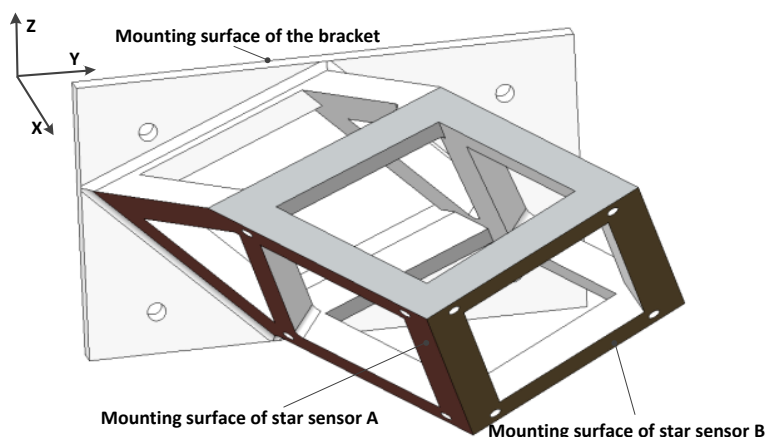

Figure 1. Model of the double star sensor bracket with complex space angle

\section{Mechanical simulation}

The finite element model of the double star-sensor bracket is shown in Figure 2.

The simulation of normal modes and frequency response adopts the same finite element model. The finite element model is modeling based on the following assumptions ${ }^{[8-9]}$ :

(1) Assuming the model is a linear elastic structure system, geometric nonlinearity, contact nonlinearity caused by the clearance in structure assembly and complex mode of damping are not considered.

(2) The connection between the star sensor and the bracket is rigid.

(3) The bracket model is discretized using tetrahedral elements.

(4) Each star sensor is simplified as a lumped point to simulate its mass.

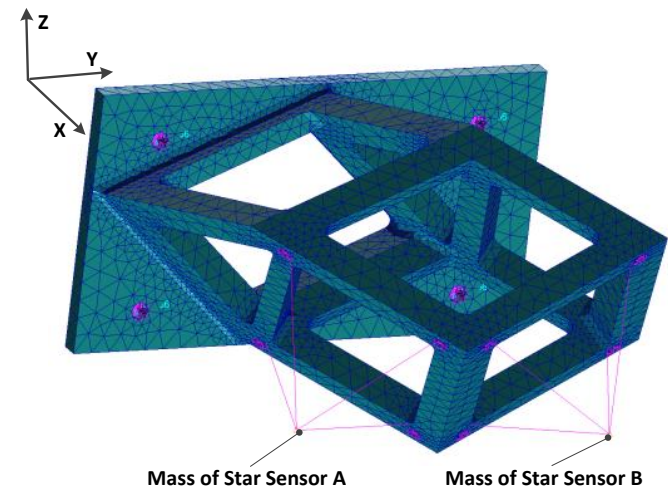

Figure 2. Finite element model of the bracket

\subsection{Normal mode analysis}

The mechanical parameters of the bracket model material are as follows: elastic modulus is $71 \mathrm{GPa}$, Poisson's ratio is 0.32 , density is $2780 \mathrm{~kg} / \mathrm{m}^{3}$. The screw holes of the mounting surface of the bracket are fixed as boundary condition. The first three modes of the model are shown in Figure 3. The first-order eigenfrequency is $317.1 \mathrm{~Hz}$, which satisfies the design requirements of the bracket stiffness.

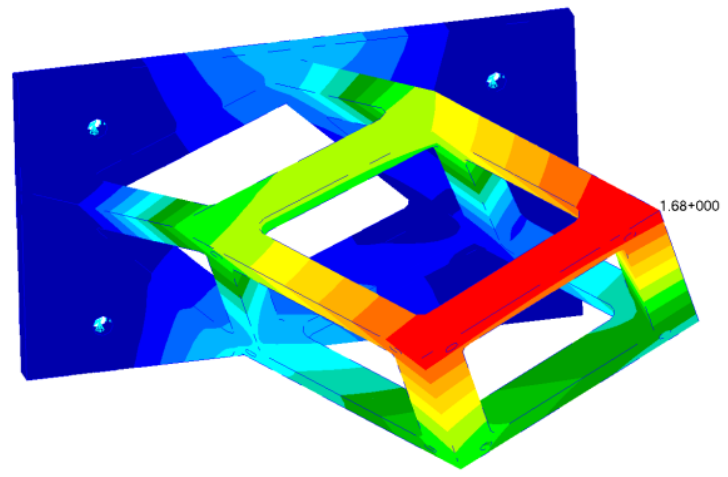

Figure 3. Mode 1: Freq. $=317.1 \mathrm{~Hz}$

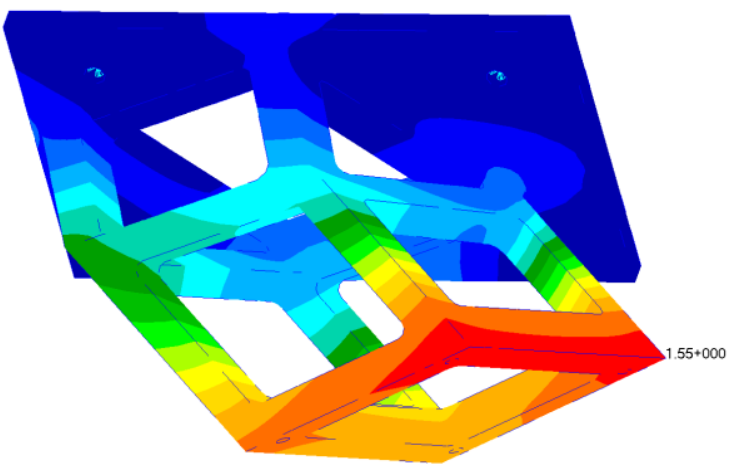

Figure 4. Mode 2: Freq. $=488.8 \mathrm{~Hz}$

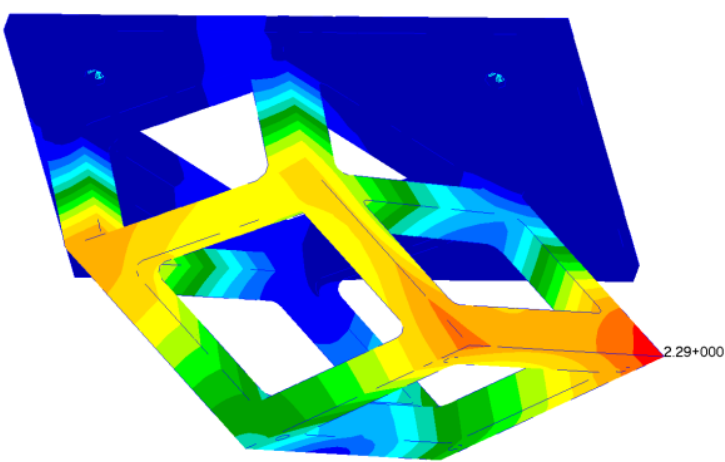

Figure 5. Mode 3: Freq. $=801.5 \mathrm{~Hz}$

\subsection{Static analysis}

According to mechanical environmental requirements of the rocket, the static loads applied along $\mathrm{X}, \mathrm{Y}$, and $\mathrm{Z}$ directions are $49 \mathrm{~m} / \mathrm{s}^{2}, 49 \mathrm{~m} / \mathrm{s}^{2}$, and $157 \mathrm{~m} / \mathrm{s}^{2}$ respectively. By static simulation, the maximum stress in the bracket is 77.4MPa, which occurs around the installation screw hole. Since the allowable stress of 2A12T4 aluminium alloy is $255 \mathrm{MPa}$ and the safety margin is 3.3 , requirements in strength are satisfied. 


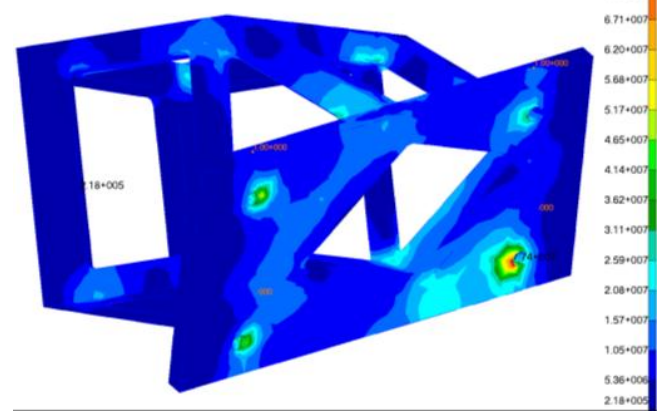

Figure 6. Von Mises Stress distribution of the bracket

\subsection{Frequency response analysis}

According to the rocket's mechanical environmental conditions, the sinusoidal response of the bracket at $0 \mathrm{~Hz}-$ $100 \mathrm{~Hz}$ is mainly investigated. Since the first-order frequency of the bracket is $317.1 \mathrm{~Hz}$, which is greater than $100 \mathrm{~Hz}$, the sinusoidal response magnification in this frequency range is less than 1.2.

According to the rocket's mechanical environmental conditions, the random vibration input for the simulation is shown in Table 2. According to simulation results, in the range of $20 \mathrm{~Hz}-2000 \mathrm{~Hz}$, the random response's RMS value magnifications of star sensor $\mathrm{A}$ in $\mathrm{X}, \mathrm{Y}$, and $\mathrm{Z}$ directions are respectively $1.27,1.66$ and 1.48 , and those of star sensor $\mathrm{B}$ in $\mathrm{X}, \mathrm{Y}$, and $\mathrm{Z}$ directions are respectively $1.47,1.86$ and 1.96 . As an example, the random vibration response curve of two star sensors in the $\mathrm{Z}$ direction is shown in Figure 7.

Table 2. Experimental conditions for random vibration

\begin{tabular}{|c|c|c|}
\hline $\begin{array}{c}\text { Random vibration } \\
\text { experiment }\end{array}$ & \multicolumn{2}{|c|}{ Power spectral density } \\
\hline Frequency $(\mathrm{Hz})$ & $\begin{array}{c}\text { Acceptance } \\
\text { level }\end{array}$ & $\begin{array}{c}\text { Identification } \\
\text { level }\end{array}$ \\
\hline $20 \sim 150$ & $+3 \mathrm{~dB} /$ oct. & $+3 \mathrm{~dB} /$ oct. \\
\hline $150 \sim 1000$ & $0.1 \mathrm{~g}^{2} / \mathrm{Hz}$ & $0.2 \mathrm{~g}^{2} / \mathrm{Hz}$ \\
\hline $1000 \sim 2000$ & $-6 \mathrm{~dB} / \mathrm{oct}$. & $-6 \mathrm{~dB} / \mathrm{oct}$. \\
\hline Root-mean-square & $11.9($ grms $)$ & $16.9($ grms $)$ \\
\hline Loading direction & $\mathrm{X} 、 \mathrm{Y} 、 \mathrm{Z}$ & $\mathrm{X} 、 \mathrm{Y} 、 \mathrm{Z}$ \\
\hline Loading time & $\begin{array}{c}1 \mathrm{~min} \text { in each } \\
\text { direction }\end{array}$ & $\begin{array}{c}2 \text { mins in each } \\
\text { direction }\end{array}$ \\
\hline
\end{tabular}

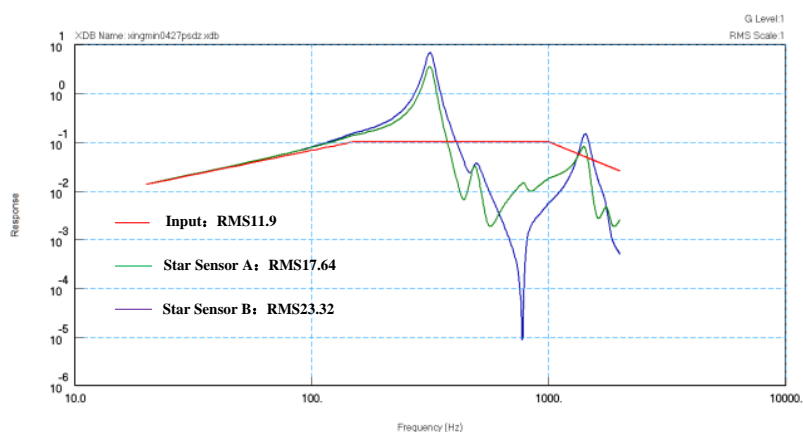

Figure 7. The simulation curve of random response of $Z$ direction

\section{Experimental verification}

In order to verify rationality of the analysis results and the structure design, meanwhile expose defects in the manufacturing process, vibration experiments were carried out $^{[10-11]}$.

The montage of the bracket and star sensors is shown in Figure 8. Sinusoidal and random vibration experiments were performed along $\mathrm{X}, \mathrm{Y}$, and $\mathrm{Z}$ directions and the results are discussed as below:

1) The first-order eigenfrequency is $308.0 \mathrm{~Hz}$. The sinusoidal response magnifications of the star sensors in $\mathrm{X}, \mathrm{Y}$, and $\mathrm{Z}$ directions are less than 1.2 for the range between $0 \mathrm{~Hz}$ to $100 \mathrm{~Hz}$. The experiments curves in the three directions of $\mathrm{X}, \mathrm{Y}$, and $\mathrm{Z}$ are respectively shown in Fig. 9-11.

2) For the range of $20 \mathrm{~Hz}-2000 \mathrm{~Hz}$, the random response's RMS value magnifications of the star sensor A in $\mathrm{X}, \mathrm{Y}$, and $\mathrm{Z}$ directions are respectively $1.69,1.79$ and 1.51 , and those of the star sensor $B$ are respectively 1.61 , 2.22 and 1.52. The measured response curves for $\mathrm{X}, \mathrm{Y}$, and $\mathrm{Z}$ directions are shown respectively in Fig. 12-14.

3) Results of precision tests after the vibration experiments are shown in Table 3. The test results show that the deviation between the star sensors and the reference is less than 30", which satisfies the design requirements.

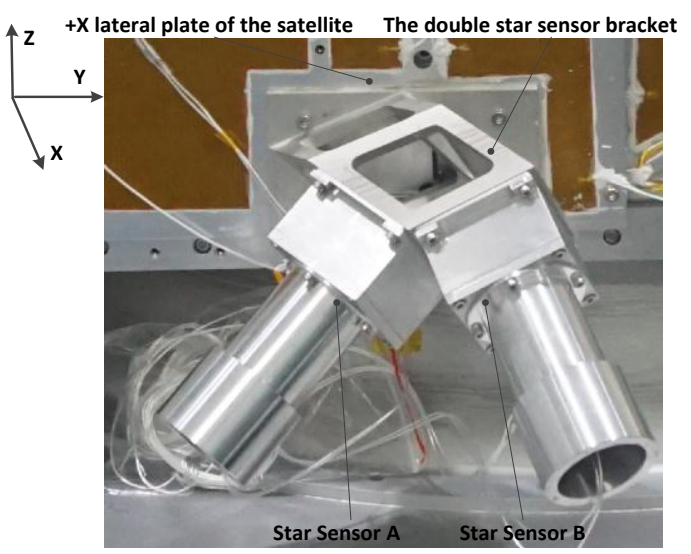

Figure 8. A montage of the combination for the bracket and star sensors 


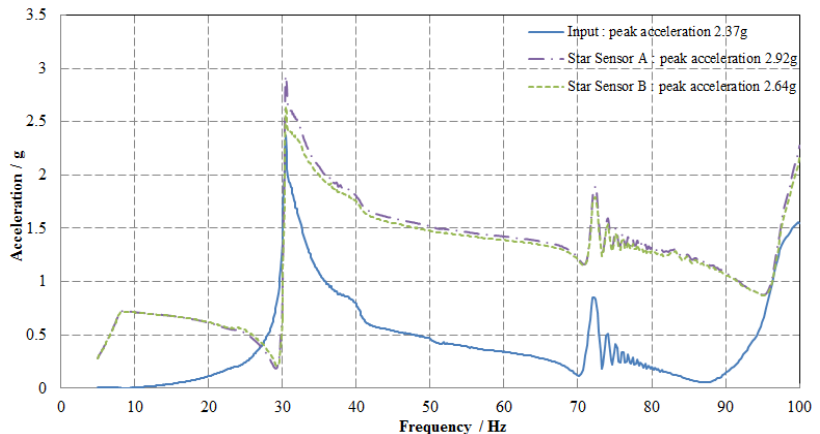

Figure 9. The sinusoidal vibration experiment curve of the bracket in $\mathrm{X}$ direction

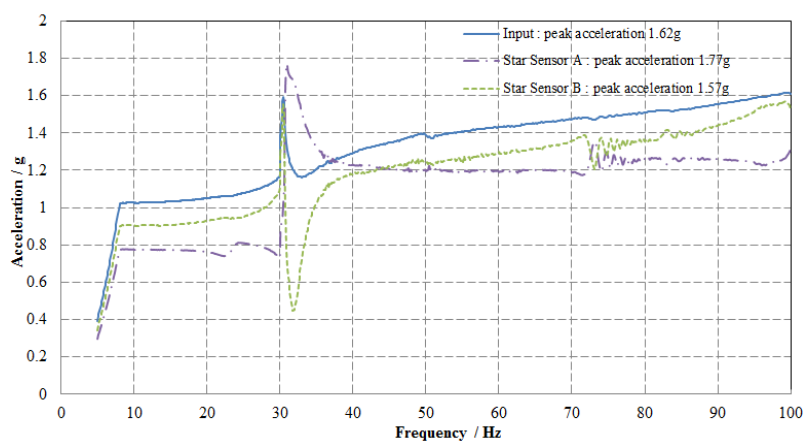

Figure 10. The sinusoidal vibration experiment curve of the bracket in $\mathrm{Y}$ direction

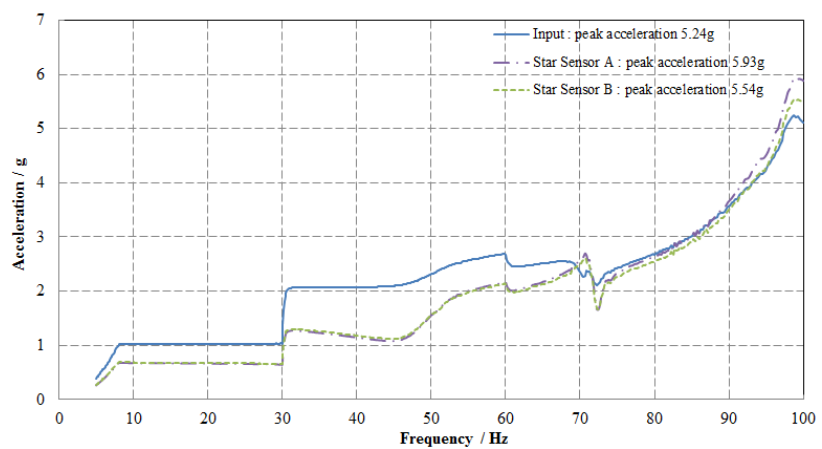

Figure 11. The sinusoidal vibration experiment curve of the bracket in $\mathrm{Z}$ direction

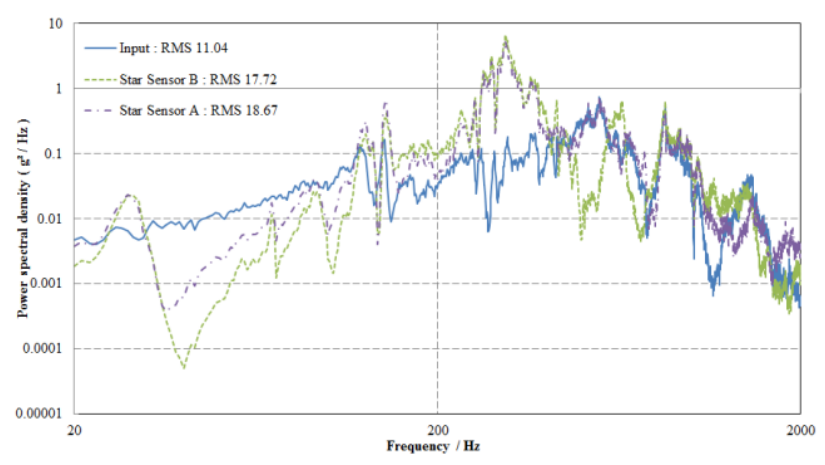

Figure 12. The random vibration experiment curve of the bracket in $\mathrm{X}$ direction

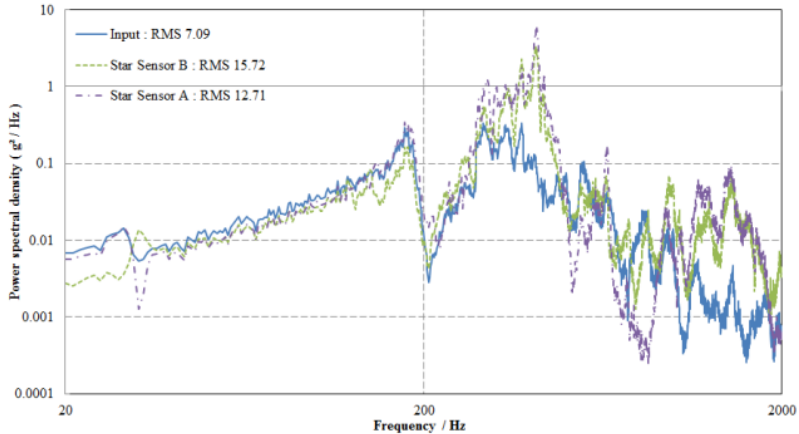

Figure 13. The random vibration experiment curve of the bracket in $\mathrm{Y}$ direction

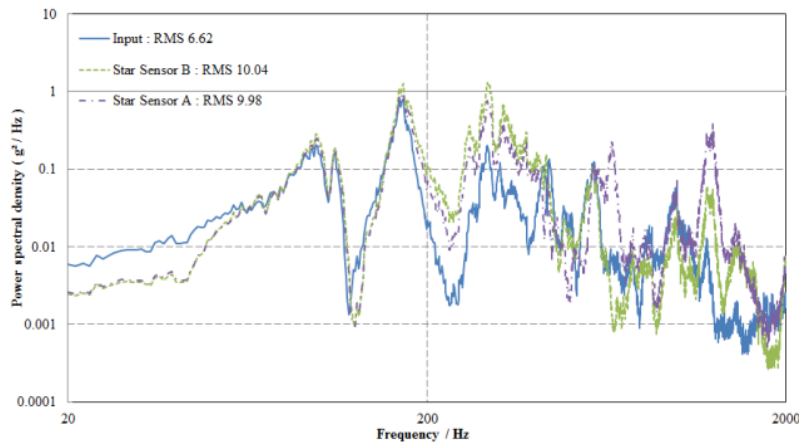

Figure 14. The random vibration experiment curve of the bracket in $\mathrm{Z}$ direction

Table 3. Results of precision tests after the vibration experiments

\begin{tabular}{|c|c|c|c|c|}
\hline \multicolumn{2}{|c|}{} & \multicolumn{3}{|c|}{ Reference prism } \\
\cline { 3 - 5 } \multicolumn{2}{|c|}{} & $\mathrm{X}$ & $\mathrm{Y}$ & $\mathrm{Z}$ \\
\hline \multirow{2}{*}{$\begin{array}{c}\text { Prism of } \\
\text { star sensor } \\
\text { A (-Y) }\end{array}$} & $\mathrm{X}^{\prime}$ & $-11.88^{\prime \prime}$ & $3.24^{\prime \prime}$ & $15.84^{\prime \prime}$ \\
\cline { 2 - 5 } & $\mathrm{Y}^{\prime}$ & $-9^{\prime \prime}$ & 0 & $-5.4^{\prime \prime}$ \\
\cline { 2 - 5 } & $\mathrm{Z}^{\prime}$ & $-12.96^{\prime \prime}$ & $7.56^{\prime \prime}$ & $-13.32^{\prime \prime}$ \\
\hline $\begin{array}{c}\text { Prism of } \\
\text { star sensor } \\
\mathrm{B}(+\mathrm{Y})\end{array}$ & $\mathrm{X}^{\prime}$ & $-20.16^{\prime \prime}$ & $1.44^{\prime \prime}$ & $24.84^{\prime \prime}$ \\
\cline { 2 - 5 } & $\mathrm{Y}^{\prime}$ & $11.16^{\prime \prime}$ & $-12.24^{\prime \prime}$ & $12.6^{\prime \prime}$ \\
\cline { 2 - 5 } & $\mathrm{Z}^{\prime}$ & $19.8^{\prime \prime}$ & $-13.32^{\prime \prime}$ & $16.56^{\prime \prime}$ \\
\hline
\end{tabular}

\section{Conclusion}

In this paper a double star sensor bracket with complex space angle is designed with aim of improving the precision of microsatellite attitude measurement and realizing mutual correction of the star sensors. Finite element simulation as well as mechanical experiments are carried out. The bracket has first-order eigenfrequency of $308 \mathrm{~Hz}$. No obvious amplification was observed for sinusoidal vibrations. The random responses as well as the precision after vibration satisfy the design requirements. 


\section{References}

1. Jorgensen J, Liebe C. The advanced stellar compass, development and operations [J]. Acta Astronautica, 1996 (11): 775-783.

2. Liao Zhibo, Fu Ruimin, Zong Xiaoyin. Optimal designing of baffle of star sensor [J]. Chinese Journal of Lasers, 2010, 37(4), 987-990.

3. Liu Haibo, Tan Jiyong, Shen Benjian. Thermal/ structure/ optical analysis of optical system of star sensor [J]. Journal of Astronautics, 2010, 31(3): 875879.

4. Tu Shancheng. Attitue dynamics and control of satellite [M]. Beijing: China Astronautics Press, 2001.

5. Huang Xin. New APS star sensor [C]. Proceedings of Annual Congress of China association for Science and Technology, 2006: 49-55.

6. Liu Lei, Zhang Lu, Zheng Xin, et al. Current situation and development trends of star sensor technology [J]. Infrared and Laser Engineering, 2007, 36(9): 25292533.

7. Wang Haiming, Zhao Hua, Yang Wentao. Mechanical analysis of a star sensor's support of ZY-1 [J]. Spacecraft Environment Engineering, 2007, 24(3): 168-173.

8. Yuan Jiajun, et al. Design and analysis of satellite structures [M]. China Astronautics Publishing House, 2004.

9. Xu Benwen, Jiao Qunying. Mechanical vibration and modal analysis fundamentals [M]. China Mechanic Industry Press, 1998.

10. Chen Zhiping, Chen Zhiyuan, Yang Shimo. Modal analysis and experiment on the main truss of space solar telescope [J]. Opto-Electronic Engineering, 2004, 31(12).

11. Xia Lijuan, Yu Yin, Jin Xianding. Experimental research and optimal design for mode characteristic of the frame structure on satellite [J]. Journal of Shanghai Jiaotong University, 2004, 38(11). 